\title{
VERIFICATION OF NUMERICAL MODEL OF FIRE AND SMOKE DEVELOPMENT IN RAILWAY TUNNEL
}

\author{
Kamila Horováa $^{\mathrm{a}}$, František Wald ${ }^{\mathrm{a}}$, Jiří Apeltauer ${ }^{\mathrm{b}}$ \\ ${ }^{a}$ Czech Technical University in Prague, Faculty of Civil Engineering, Prague, Czech Republic \\ ${ }^{\mathrm{b}}$ Brno University of Technology, Institute of Road Structures, Brno, Czech Republic
}

\begin{abstract}
Simulation of fire spread and development of toxic gases during a fire accident in a railway tunnel allows prepare and validate models of safe evacuation of people. Highly complex problem of fire dynamics in a tunnel can be solved by the aid of numerical models based on CFD method. In order to check the quality of prediction models the procedure of verification is used. A relatively simple model of a single track railway tunnel is solved in two independent codes - FDS and Smart Fire. Accuracy of the model prediction is verified by the aid of gas temperature resolution along the tunnel length. To estimate an error based on different mesh resolutions of numerical model, calculation of the same model is carried out using different mesh density.
\end{abstract}

Keywords: verification, FDS, Smart Fire, railway tunnel, fire and smoke development

\section{INTRODUCTION}

Nowadays, mathematical modeling of fire dynamics plays a key role in fire engineering. Together with the development of computer technology, a number of sophisticated software tools, which implement the so-called Computational Fluid Dynamics method (CFD) dramatically increases. Despite the rapid software development there are many contradictory opinions about the reliability of computer predictions. It is almost impossible to model all the aspects of a complex event, yet valuable conclusions from a series of simulations can be concluded if proper tools and statistical measures are used through the verification and validation $(\mathrm{V} \& \mathrm{~V})$ procedures (Kwasniewski and Bojanowski, 2013).

Today $\mathrm{V} \& \mathrm{~V}$ is recognized as the primary method for evaluating the confidence of computer simulations. Verification usually uses comparison of computational solutions with highly accurate (analytical or numerical) benchmark solutions and among themselves. Verification is supposed to deliver evidence that mathematical models are properly implemented and that the numerical solution is correct with respect to the mathematical model. Due to the high complexity of problems that are practically important, such verification can be conducted only using approach where the reasoning is based on the experience coming from repeated calculations. A standard example is the error estimation based on numerical results of for different mesh resolutions. Numerical solutions applied for verification can represent mathematical models with little physical importance. The problem considered should be relatively simple (Kwasniewski and Bojanowski, 2013). Despite the before mentioned, in (ASME, 2006) it has been pointed out that even the most extensive verification cannot remove all errors.

Simulation of fire spread and development of toxic gases during a fire accident in a rail tunnel allows prepare and validate models of safe evacuation of people. Highly complex problem of fire dynamics in a tunnel can be solved by the aid of CFD method, using for example code FDS [1]. It can simulate the temperature resolution and toxic gases development in a tunnel, which can be together with visibility considered as the most important parameters affecting the safety of persons involved in tunnels accidents. In order to check the quality of prediction models the procedure of verification is used. 


\section{NUMERICAL MODELLING}

During a fire in a tunnel there are three-dimensional flows which are influenced by buoyancy effects. In addition, the flow velocity and length scales are sufficiently large that the flow is generally turbulent. At the fire, a combustion process takes place, and so there are chemical reactions going on, producing soot particles and combustion products at high temperatures (Rhodes, 2012). Part of the heat is transferred by radiation and part by convection to the tunnel walls. It is possible to formulate models to predict the growth rate of a fire, although a complete knowledge of the properties of the reacting material is required. CFD models can provide a framework for including all these phenomena in a calculation. The computer programs Fire Dynamics Simulator (McGrattan et al, 2007) and Smart Fire (Ewer et al, 2013), which are frequently used representatives of CFD fire modeling, are used in this study to verify the accuracy of the model of fire and smoke development in railway tunnel.

\subsection{Model of a tunnel}

In order to keep the problem relatively simple, a part of model of a single-track railway tunnel is used. The model consists of simple geometry of the tunnel length of $50 \mathrm{~m}$ and a rectangular cross section with dimensions of $5.0 \times 5.0 \mathrm{~m}$. Computational domain consists of three meshes with a division of $200 \times 20 \times 20$ (denoted as coarse grid). The fire outbreak of constant power of $1 \mathrm{MW}$ and dimensions of $2.0 \times 1.0 \times 0.5 \mathrm{~m}$ is located in the middle of the tunnel length (in FDS code an obstacle with surface of specified heat release rate per unit area HRRPUA $=500 \mathrm{~kW} / \mathrm{m}^{2}$ ). The tunnel consists of absolutely non-conductive material (in FDS code adiabatic surface, layer thickness of $0.1 \mathrm{~m}$ ). Tunnel portals opened throughout the cross-sectional area provide natural gas flow (in FDS code plane surface type VENT is OPEN). Before activating the burner, the inner tunnel environment is set, the initial gas temperature is $15^{\circ} \mathrm{C}$ (in FDS code as TMPA). In the tunnel there are no flammable materials. The total simulation time is $150 \mathrm{~s}$. Gas temperature and gas velocity are calculated in both codes, FDS and Smart Fire, in the axis of the tunnel cross section at a height of $0.55 \mathrm{~m}$ under the ceiling. In FDS turbulence model is applied by Smagorinski formulas of large eddy simulation (LES) with coefficient $C_{s}$ equals to 0.2. In contrary Smart Fire uses RANS-based model of two equations' K-epsilon model to solve turbulence flows. In both software reaction of burning is kept in default settings (in FDS mixed-fraction model of propan). Heat transfer by radiation is applied by 100 discrete angles in FDS, in Smart Fire by 24-rays model.

\section{$2.2 \quad$ Results}

Graphical visualization of hot gases flow and gas velocity on a plane located in the middle of the tunnel width coming from the computing code FDS is shown in Fig. 1 and Fig. 2. Considering the same boundary conditions at both portals of the tunnel and symmetrically located fire outbreak, the layer of smoke and hot gases travels nearly uniformly in both directions. In Figs. 1a to 1c a border of $80^{\circ} \mathrm{C}$, which is assumed as critical for escaping persons, is highlighted. From the figures it can be seen that the thickness of hot gas layer below the ceiling between $30 \mathrm{~s}$ to $100 \mathrm{~s}$ does not grow significantly due to sufficient supply of cool air in a relatively short tunnel. Gases with higher temperature gradually move further away from the centre of the burner to the tunnel portals. Thoroughly looking on Figs. $2 a-2 b$, inflow of the fresh air towards the fire outbreak, may be observed. In addition to the gas temperature and gas flow velocity visibility, content of toxic substances in the combustion products or temperature of the lining structure can be determine from the numerical calculation.

Results of gas temperature of the computing codes FDS and Smart Fire detected by a linear sensor placed in the axis of the tunnel cross section at the height of $0.55 \mathrm{~m}$ under the ceiling in $30 \mathrm{~s}$ and $100 \mathrm{~s}$ are shown in Fig. 3. As it could be predicted the highest temperature is directly above the burner. Getting farer from the burner, temperature decreases. Despite the expectation, observed temperature at region between the burner and the part of the tunnel where one dimensional flow dominates (about 5 meters in both directions from the axis of the burner) is too low. This may be 
influenced by high velocity and high temperature gradients in this region. Using relatively coarse mesh, described characteristics cannot be captured.

Neglecting the area affected by direct influence of the burner, hot gas layer under the ceiling is below $100{ }^{\circ} \mathrm{C}$. There is not big difference between values reached in $30 \mathrm{~s}$ and $100 \mathrm{~s}$. Temperature is getting lower with increasing distance from the burner.

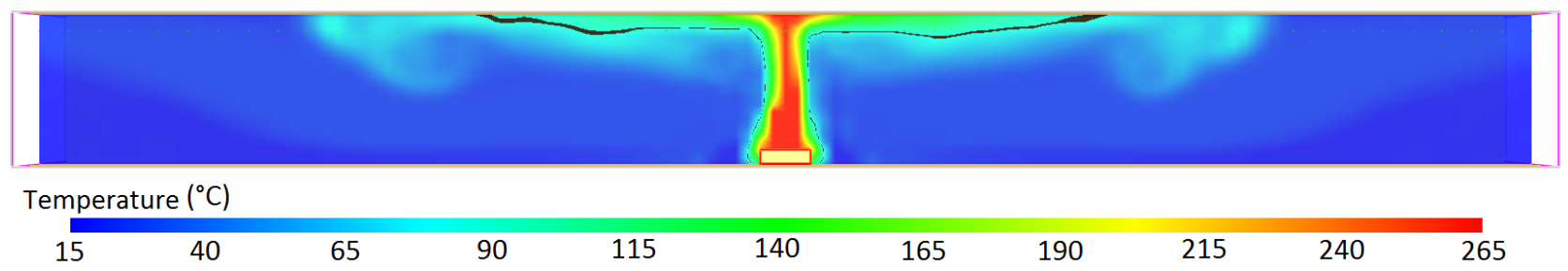

a)

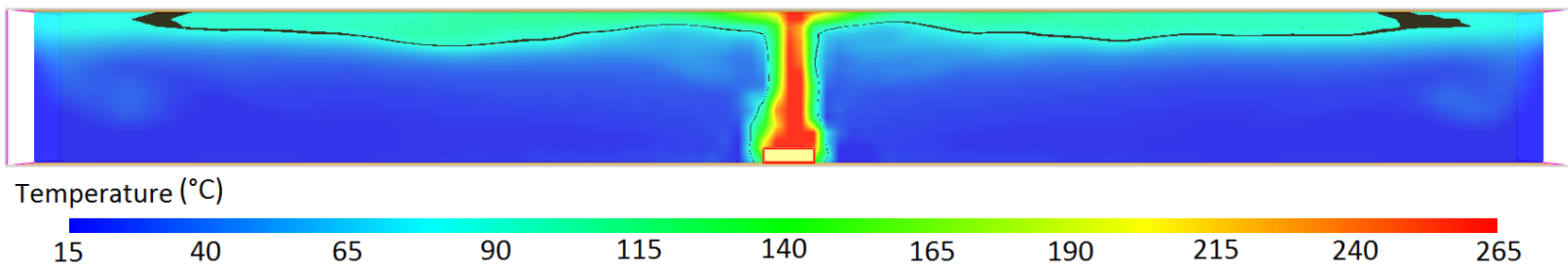

b)

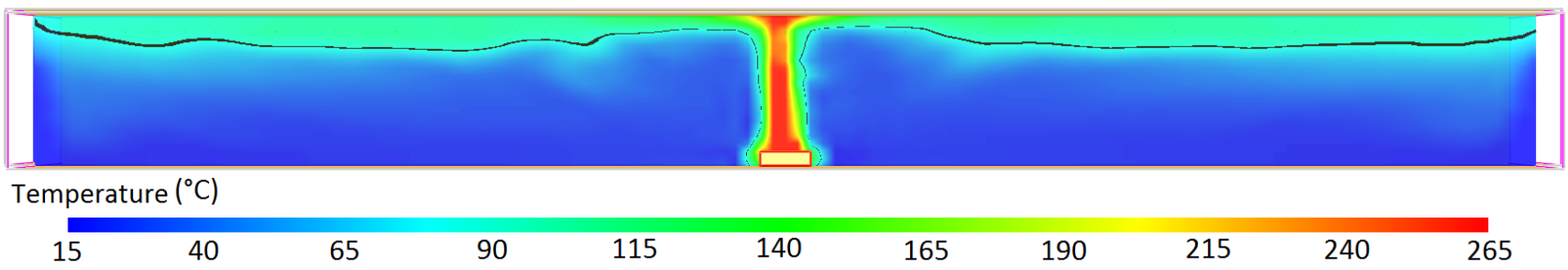

c)

Fig. 1 Visualization of development of hot gases: a) in $12 \mathrm{~s}, \mathrm{~b}$ ) in $30 \mathrm{~s}, \mathrm{c}$ ) in $100 \mathrm{~s}$

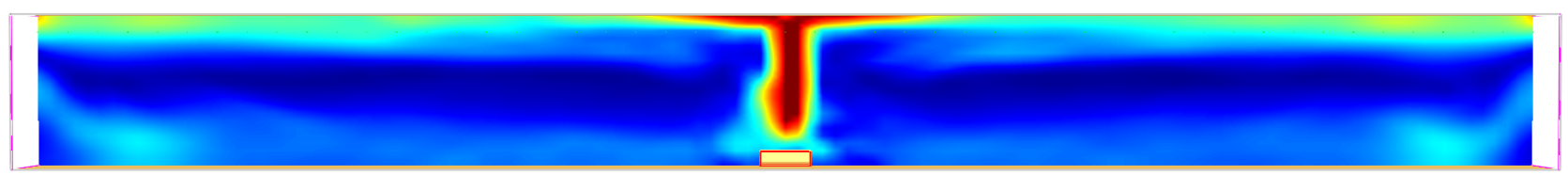

Velocity $(\mathrm{m} / \mathrm{s})$

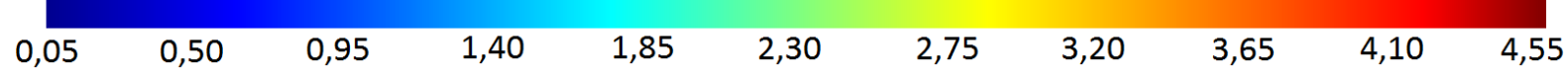

a)

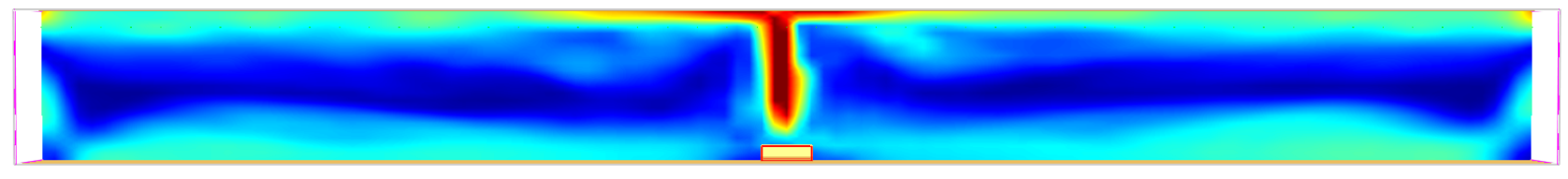

Velocity (m/s)

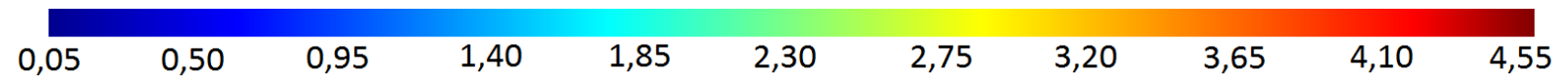

b)

Fig. 2 Visualization of gas flow velocity: a) in $30 \mathrm{~s}$, b) in $100 \mathrm{~s}$ 


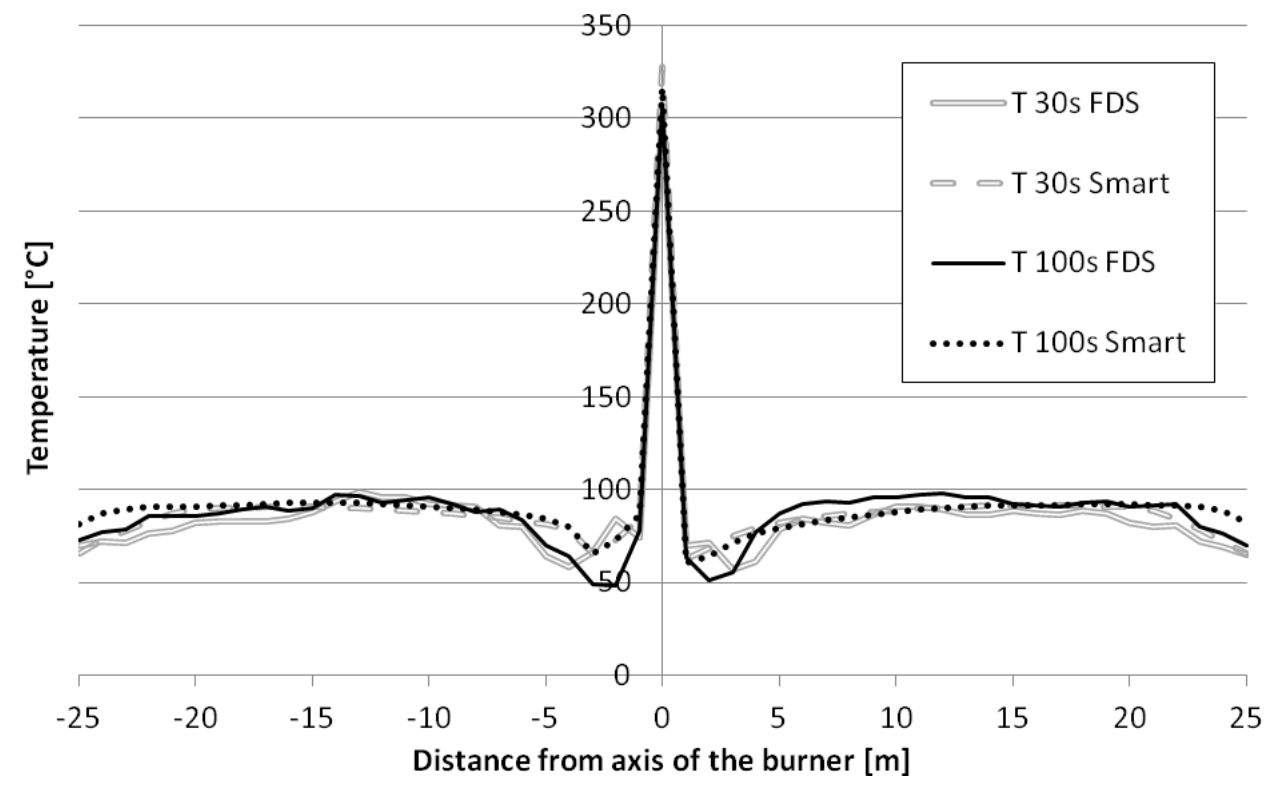

Fig. 3 Gas temperature distribution along the tunnel length in $30 \mathrm{~s}$ and $100 \mathrm{~s}$ (origin of $\mathrm{x}$ axis lays in axis of the burner)

\section{VERIFICATION}

In order to verify the model of hot smoke development in railway tunnel, gas temperature of the computing codes FDS and Smart Fire detected by a linear sensor placed in the axis of the tunnel cross section at the height of $0.55 \mathrm{~m}$ under the ceiling in $30 \mathrm{~s}$ and $100 \mathrm{~s}$ are compared. From the ratio of calculated values of the gas temperature (FDS / Smart Fire) along the tunnel length in Fig. 4 it can be seen that the maximum difference in the area affected by flame ( 5 meters in both directions from the axis of the burner) is about $20 \%$. In the region of dominant one dimensional gas flow, farer from the axis of the burner, the values differ up to $10 \%$. According to the theory of probability and statistics data can be described by the arithmetic mean, mean square error and standard deviation in time $30 \mathrm{~s}$ (individual values in the forenamed order are $0.96 ; 0.01 ; 0.08$ ) and at the time of $100 \mathrm{~s}(0.97,0.01,0,10)$. Paerson's correlation coefficient is equal to 0.978 in time 30 $\mathrm{s}$ and at time $100 \mathrm{~s}$ it equals to 0.970 . Results of statistical relationships demonstrate that the verification of the numerical model in both software has reached a good agreement.

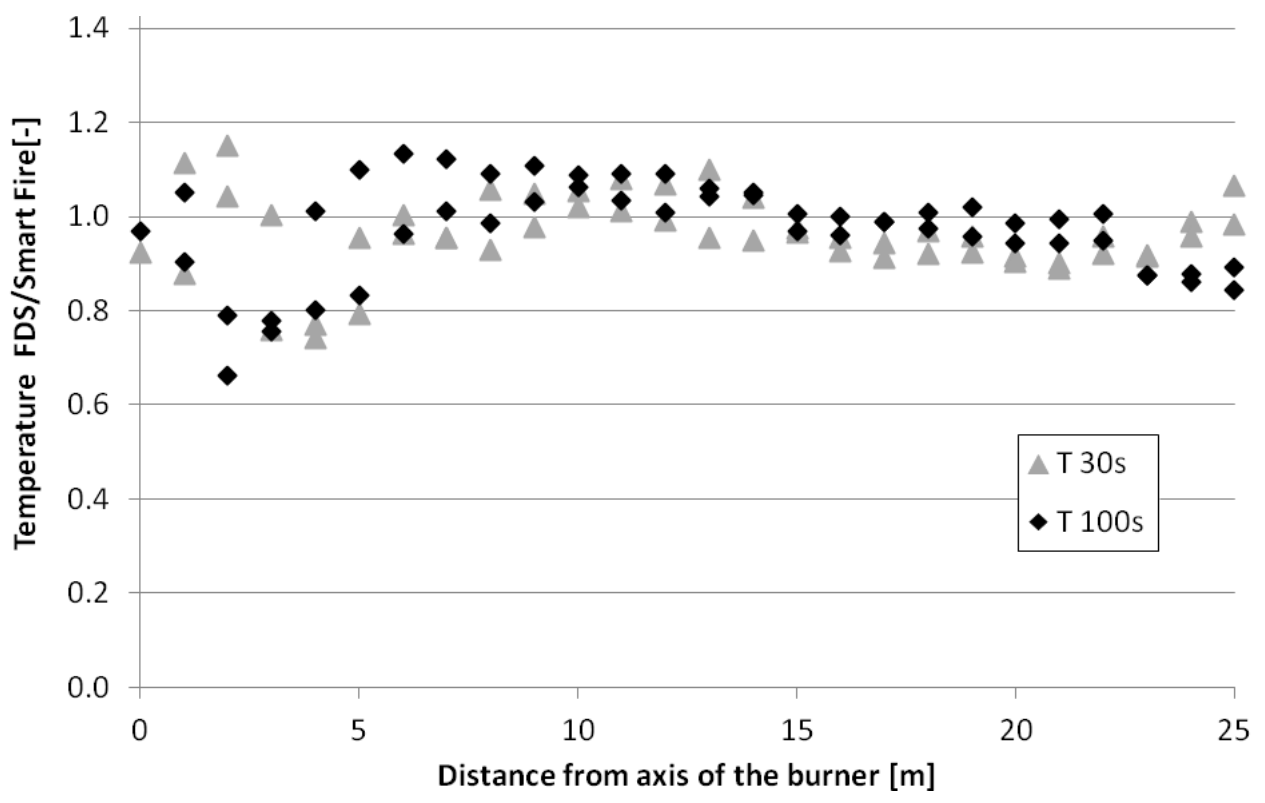

Fig. 4 Verification of gas temperature in $30 \mathrm{~s}$ and $100 \mathrm{~s}$ 


\section{SENSITIVITY STUDY}

To estimate an error based on different mesh resolutions of numerical model, calculation of the same model described in chapter 2.1 is carried out using different mesh density. In this case finer division of $400 \times 40 \times 40$ is applied to all meshes. Then, the size of grid cell is $0.125 \mathrm{~m}$. In Fig. 5 results of gas temperature resolution in $30 \mathrm{~s}$ and $100 \mathrm{~s}$ is shown. The temperature is calculated in the same position of $0.55 \mathrm{~m}$ below the tunnel ceiling. Comparing to Fig. 3, only one symmetrical part of the tunnel is presented. Looking at the maximum temperature above the burner, $100{ }^{\circ} \mathrm{C}$ higher temperatures are reached by the model with finer mesh. In the part of the tunnel where one dimensional flow dominates, temperature oversteps $100{ }^{\circ} \mathrm{C}$ comparing to the model with coarse mesh. The biggest influence of the application of finer mesh is visible in the region between the burner and the part of the tunnel where one dimensional flow dominates. Above described fall of the temperature disappeared, and the results look more reliable.

Fig. 6 illustrates the influence of grid size on gas temperature in above described regions. In the region with dominant one dimensional flow the temperature rise of the model with finer mesh is about $20 \%$. However temperatures in the most affected field differ up to $100 \%$.

\section{CONCLUSIONS}

Correctly applied CFD models may reproduce the qualitative behaviour of fire in a tunnel. The degree, to which qualitative agreement is achieved, is necessary to find out by the aid of verification and validation process. Verification study presented in this paper shows a relatively simple model of a single track railway tunnel solved in two independent codes - FDS and Smart Fire. Results of statistical relationships demonstrate that the verification of the numerical model reached a good agreement (Paerson's correlation coefficient is equal to 0.97). To estimate an error based on different mesh resolutions of numerical model, calculation of the same model is carried out using different mesh density. The undertaken sensitivity study shows that application of the relatively coarse mesh in regions influenced by high velocity and high temperature gradients does not lead to reliable results. It is therefore recommended to apply finer mesh in the region adjacent to the burner. Whereas the region where one dimensional flow dominates, coarser mesh may be used to reduce calculation time.

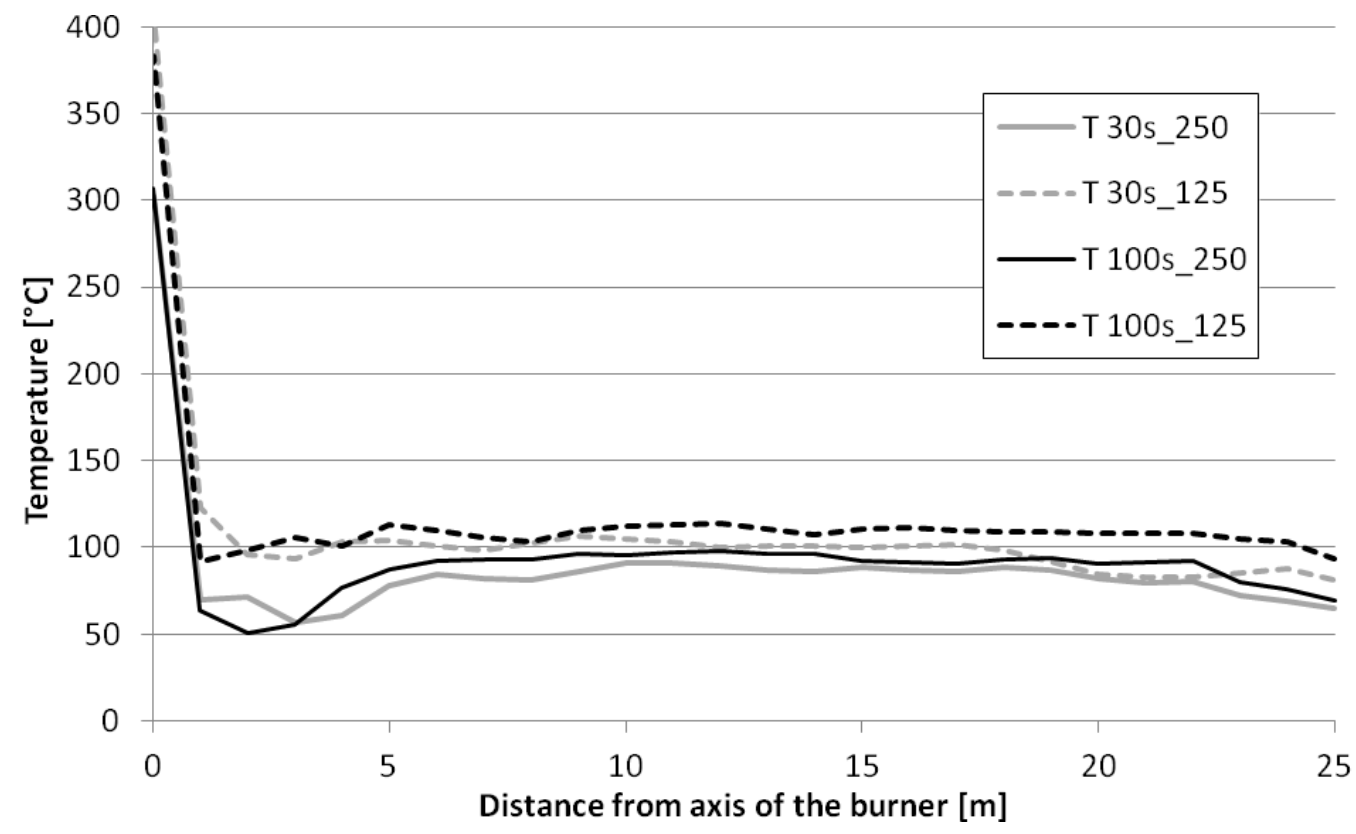

Fig. 5 Influence of grid size on gas temperature resolution in FDS model 


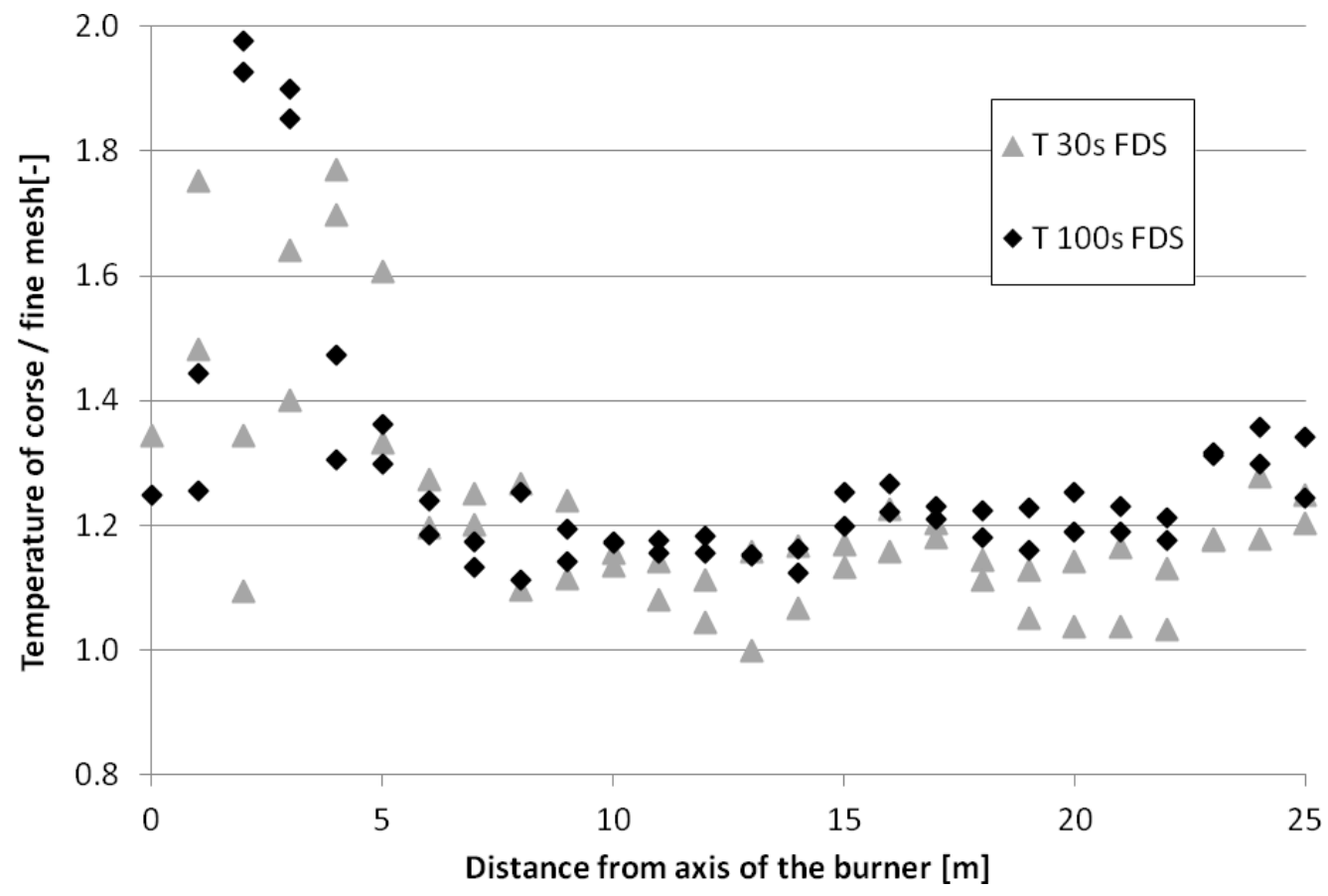

Fig. 6 Verification of grid size influence on gas temperature resolution in $30 \mathrm{~s}$ and $100 \mathrm{~s}$

\section{REFERENCES}

ASME, 2006. Guide for Verification and Validation in Computational Solid Mechanics, The American Society of Mechanical Engineers, ISBN 079183042X.

Beard, A., Carvel, R., et al. 2005. Handbook of Tunnel Fire Safety, ICE Publishing, Second edition, London, UK.

Ewer J., Jia F., Grandison A., Galea E., Patel M., 2013. Smart Fire - User guide and technical manual, Smartfire tutorials.

ISO FDIS 16730, Fire safety engineering - Assessment, verification and validation of calculation methods, 2008.

Kwasniewski, L., Bojanowski, C., 2013. Principles of Verification and Validation, Proceedings of International Conference Applications of Structural Fire Engineering, 19-20 April, 2013, Prague, Czech Republic, p. 13-22.

McGrattan K., Hostika S., Floyd J., Baum H., Rehm R., 2007. Fire Dynamics Simulator (Version 5), Technical Reference Guide, NIST Special Publication 1018-5, p. 86, October 2007, U.S. Government Printing Office, Washington.

Rhodes, N., 2012. CFD modelling of tunnel fires, in Handbook of Tunnel Fire Safety, ICE Publishing, London, UK, p. 329-346.

Wald F., Burgess I., Kwasniewski L., Horová K, Caldová E., 2014a. Benchmark studies, Experimental validation of numerical models in fire engineering, CTU Publishing House, Czech Technical University in Prague.

Wald F., Burgess I., Kwasniewski L., Horová K., Caldová E., 2014b. Benchmark studies, Verification of numerical models in fire engineering. CTU Publishing House, Czech Technical University in Prague. 